\title{
Estabilidad Epistemológica del Profesor Debutante y Espacio de Trabajo Matemático
}

\author{
Nobel Teacher's Epistemological Stability and Mathematical Working \\ Space
}

\author{
Elizabeth Montoya-Delgadillo* \\ Jaime Mena-Lorca \\ Arturo Mena-Lorca ${ }^{* * *}$
}

\begin{abstract}
Resumen
En este trabajo estudiamos la estabilidad epistemológica de profesores debutantes. Para ello, hemos hecho un estudio cualitativo de su ETM-idóneo en el momento en que desarrollan un dominio matemático con sus estudiantes, de modo de clarificar los elementos que ponen en juego cuando despliegan una tarea en el aula. Mostramos resultados relativos al favorecimiento eventual que hacen esos profesores de la concreción de las génesis semiótica, instrumental y discursiva, y de la circulación entre los distintos polos de los planos epistemológico y cognitivo. Del estudio se desprende que la epistemología del profesor debutante no es estable, debido a una tensión entre su ETM-personal y su ETM-idóneo.
\end{abstract}

Palabras clave: Estabilidad Epistemológica. Profesores Debutantes. Algebrización.

\begin{abstract}
In this research, we study the epistemological stability of beginning teachers. To do this, we have made a qualitative study of their suitable-MWS when developing a mathematical domain with the students, so as to clarify the elements that they put into play when unfolding a task in the classroom. We show results related to the eventual favoring that those teachers make of the realization of semiotic, discursive and instrumental genesis, and of the circulation between the different poles of the epistemological and cognitive planes. The study shows that the epistemology of beginning teachers is not stable, due to tension between their personal-MWS and their suitable-MWS.
\end{abstract}

Keywords: Epistemological Stability. Beginning Teachers. Algebrization

\footnotetext{
* Doctor en Didáctica de la Matemática, Paris Diderot. Profesora Pontificia Universidad Católica de Valparaíso (PUCV). Av. Brasil 2950, casilla 4059,Valparaíso, Chile. elizabeth.montoya @ pucv.cl

** Doctor en Matemática, Universidad de Iowa. Profesor Pontificia Universidad Católica de Valparaíso (PUCV). Av. Brasil 2950, casilla 4059,Valparaíso, Chile. jaime.mena @ pucv.cl

*** Doctor en Didáctica de la Matemática, CICATA-IPN. Profesor Pontificia Universidad Católica de Valparaíso (PUCV) \& CIAE. Av. Brasil 2950, casilla 4059,Valparaíso, Chile. arturo.mena@ pucv.cl
} 


\section{Introducción}

Esta investigación se inscribe en la teoría del Espacio de Trabajo Matemático, ETM (KUZNIAK, 2011), que en sus inicios fue conocida como teoría de Paradigmas y Espacio de Trabajo Geométrico (HOUDEMENT; KUZNIAK, 1996; 2006).

Llamamos Profesor Debutante, PD, a uno que lleva a lo sumo dos años de ejercicio profesional. Ahora bien, siguiendo a (PORLÁN; RIVERO; DEL POZO, 1998), entendemos por epistemología del profesor las creencias que él manifiesta sobre el conocimiento, y su construcción y transmisión en el contexto escolar; para nosotros, tales creencias tienen un carácter paradigmático, y la construcción es la del objeto matemático en cuestión -y la transmisión es una metáfora.

Reportamos acerca de la estabilidad de esa epistemología al cambiar el PD su rol de aprendiz de la matemática y de su enseñanza por uno de profesor de matemática en ejercicio; para ello, analizamos la expresión de su ETM-idóneo en el aula, cuando desarrolla un dominio matemático y propone y aborda tareas.

Nos hemos preguntado, en primer lugar, si, en su ejercicio docente, los PD utilizan estrategias que se ocupen de cada uno de los tres polos que constituyen cada uno de los planos horizontales, epistemológico y cognitivo, que explicita la teoría y, más precisamente, si acaso esos profesores fomentan cada una de las génesis -semiótica, instrumental y discursiva- y, aún más, la circulación entre los planos verticales. (KUZNIAK; RICHARD, 2014).

Así, hemos clarificado los elementos del ETM-idóneo puestos en juego por el profesor cuando propone una tarea en un dominio específico, y determinado el rol de objetos de otros dominios en las génesis activadas por esa tarea.

$\mathrm{Al}$ respecto, nos hemos preguntado: cuando se utiliza ecuaciones en el dominio de la geometría (y de las probabilidades, etc.), ¿es la ecuación solo un artefacto simbólico en la geometría o puede la aparición de aquellas comportar un cambio de dominio -en el sentido de que la tarea geométrica se convierta en una puramente algebraica y no retorne al dominio de origen-?

En general, lo anterior se relaciona tanto con identificar con claridad el dominio de trabajo como con el estudio en profundidad de las génesis que activa el PD en el aula (MONTOYA-DELGADILLO; MENA-LORCA; MENA-LORCA, 2014) en dominios específicos: análisis, álgebra, geometría, probabilidades. 
Para estudiar la estabilidad epistemológica, observamos durante un año a 6 PD chilenos de secundaria, todos pertenecientes a distintos establecimientos, y realizamos 10 grabaciones en video, en sesiones de clase de 1,5 horas cada una; además, se entrevistó a algunos de ellos.

Estudios tales como (OECD, 2004; UNESCO, 2013) señalan que, en general, los profesores chilenos no logran contribuir de manera significativa a erradicar los males mayores del sistema, y los procesos de inducción de profesores debutantes frustran la innovación que ellos procuran realizar a partir de su formación inicial. (OECD, 2004).

\section{EI ETM de profesores debutantes}

\subsection{El marco teórico}

El constructo teórico del ETM nos provee de una perspectiva para analizar al PD en tres dimensiones diferentes de su actuar. Por una parte, cómo trabaja con la matemática, cómo se comporta él como matemático: su ETM personal. Por otra, en cuanto profesor en su concepción de la matemática que debe enseñar, en el proceso de transposición de esta para elaborar propuestas didácticas efectivas en la construcción de saberes matemáticos y las reorganizaciones que esa transposición comporta en su rol de profesor: su ETM idóneo. En aquel actuar incide además, naturalmente, el espacio de trabajo constituido por lo que la comunidad sustenta, lo que esta entiende por actividad y por corpus matemáticos: el ETM de referencia (KUZNIAK, 2011; KENIA; RICHARD, 2014). Lo anterior, nos permite focalizarnos en la matemática y observar su desarrollo en cuanto a su relación con el sujeto que la está construyendo o ayudando a construir.

Ahora bien, un PD, el aspecto paradigmático es relevante, incluso más que para un alumno en su práctica profesional de profesor, según describe (MONTOYA-DELGADILLO, 2014) en lo que llamó las transiciones. Bajo el paradigma de la institución (liceo) a la que se incorpora, el cual no reconoce corresponsabilidad del aprendizaje, el PD enfrenta la compleja tarea de ser en su aula el único garante de ese aprendizaje. De hecho, la noosfera presiona al profesor centrando la crítica a la calidad de la educación en que este está realizando mal su tarea ya sea por desidia o por falta de recursos de enseñanza (MONTOYA-DELGADILLO, 2014). 


\subsection{La epistemología del PD}

Es claro que la epistemología del PD está influida por su formación inicial, recientemente concluida, y por la comunidad que lo recibe como profesional.

La primera aporta con los cursos de matemática, de didáctica y de pedagogía, $\mathrm{y}$, en buena medida, con las prácticas profesionales. La segunda le suministra, entre otras cosas, planes y programas, textos escolares, proyecto educativo y formas en que se plasman esos elementos en las asignaturas de matemáticas del establecimiento. Sus profesores formadores aportaron información (saberes) para su aproximación al ETM-referencia, y cierta noción respecto de qué ETM-idóneo debe desarrollar cuando egrese.

De tal manera, el ETM-idóneo del PD está tensionado por los elementos que la institución formadora implícitamente le propuso y los que percibe en la institución que lo acoge como profesor. Esta última podría catalizar aquellas nociones y saberes, pero a menudo tiende a modificarlos en forma substantiva y aun, a ignorarlos. (Ver, por ejemplo, (MONTOYA-DELGADILLO; VIVIER, 2014).

Hay una tensión adicional en la epistemología del PD, originada por las exigencias que el propio profesor se hace en relación al ETM-personal que cree que debe desarrollar para responder a las exigencias de la matemática; tales exigencias repercuten, naturalmente, en su ETM-idóneo.

En efecto, el conjunto de los matemáticos que participan en su formación inicial exige al estudiante (la construcción de) un ETM-personal en el cual todas las génesis estén activas y todos los polos de los planos estén articulados. Por lo demás, y a pesar de la necesaria transposición que efectúan de sus respectivos saberes, aquellos se sitúan en el paradigma 'más avanzado' de cada uno de estos: el representamen suele consistir solo de símbolos abstractos, los artefactos aceptados son exclusivamente los validados por la comunidad matemática, el proceso de prueba está sustentado explícitamente por la lógica formal. Naturalmente, todo esto influye en (el intento de) la articulación de los planos cognitivo y epistemológico, vía las génesis, que hace el PD para construir su ETM-personal, y forma parte fundamental de su epistemología, ya que aquella es la matemática y aquella la forma de hacer matemática que le ha sido enseñada y que en alguna medida ha experimentado. Es también natural esperar que la falta de claridad en cuanto a distinguir el ETM-personal del ETM-idóneo se traduzca en errores de apreciación del PD en cuanto a su rol. 


\subsection{La estabilidad epistemológica}

En el PD coexisten principalmente (y en transición) el ETM-personal de su formación inicial y el ETM-idóneo como profesional en formación. La epistemología del PD evoluciona en estos términos y la pregunta acerca de su estabilidad refiere al cambio que experimenta en relación con el idóneo que se expresa en los perfiles de egreso de la institución formadora: el profesional será capaz de transponer adecuadamente los saberes para que sus alumnos construyan matemática; es decir, que las propuestas didácticas del PD permitirán a los alumnos activar las génesis y lograr circulaciones que desarrollen plenamente los distintos dominios de las matemáticas que el currículo exige. (MONTOYA-DELGADILLO et al., 2014).

Lo anterior requiere que el profesor en formación aprenda construcciones propias de los dominios matemáticos que son de su competencia y que sepa identificar las distintas visualizaciones del representamen, que pueda instrumentalizar los artefactos y que tenga la capacidad de justificar los resultados que utiliza en las pruebas o en los procesos de instrumentalización.

Al respecto, (MONTOYA, 2014) da evidencia de que el ETM-idóneo del profesor en la formación inicial muestra una debilidad en la transposición de la demostración en geometría, es decir, que desde ya es difícil esperar un idóneo del profesional que active la génesis discursiva y logre una circulación apropiada entre el referencial y la prueba.

Esto último sugiere la necesidad de observar a los PD en el aula, ya que, por una parte, el discurso del ETM-personal del profesor tiene injerencia en su ETM-idóneo, y, por otra, debido a su formación, él cuenta con herramientas de otros dominios que pueden surgir en forma inapropiada en el proceso de construcción del conocimiento en el dominio específico.

\section{Aproximación metodológica}

Precisamos primero que, desde 2013, el sistema escolar chileno tiene 6 años de Enseñanza Básica (primaria, hasta la edad de 11-12 años) y 6 de Enseñanza Media (secundaria, desde los 13-14 años). En la Enseñanza Media hay cuatro ejes temáticos: Números, Álgebra y funciones, Geometría, y Probabilidad y estadística. El sistema distingue al liceo del colegio, teniendo este último financiamiento particular; aquí no haremos esa distinción. 
Nuestras indagaciones y evidencias se enmarcan, según lo explicitado en la Introducción, en un seguimiento por un año a seis PD en aulas, esto es, a profesores con a lo sumo dos años de servicio, en su actividad de clases, como parte de un proyecto en el cual nos preguntamos por las concepciones matemáticas desarrolladas en el aula de liceo en el sistema educativo chileno.

En el periodo 2011-2012, realizamos 10 grabaciones en video, en sesiones de clase de 1,5 horas cada una; además, entrevistamos a algunos de los PD. Luego, clasificamos esas sesiones según los contenidos para determinar el dominio utilizado en cada caso. Posteriormente hemos identificado episodios y analizado las tareas y las circulaciones desplegadas por esos profesores.

Reportamos aquí dos casos entre los PD observados, que consideramos relevantes por lo que manifiestan del ETM-idóneo: PD3 es representativo de la mayoría de los PD, y, PD5 es excepcional por ser el único que activa todas las génesis.

El contenido desarrollado por PD3 es la Regla de Laplace, del eje Probabilidades, en el nivel 6; el de PD5 es resolución de ecuaciones lineales, del eje Álgebra, en el nivel 4, ambos de enseñanza media.

\section{Algunas evidencias}

\section{1 "Algebrización" de los dominios}

Como se sabe, la algebrización que se observa en diversos dominios matemáticos es un recurso habitual y poderoso en la matemática. Cuando un especialista recurre al álgebra tiene claridad del rol que cumple esa en el dominio en que trabaja. De análoga manera, cuando un profesor del sistema, en su tarea de desarrollar en forma clara y en lo posible plena un dominio del currículo a su cargo, recurre al álgebra, debería ocuparse de situarla en un rol instrumental; pero ese no parece ser el caso.

En efecto, los resultados de las grabaciones y el desarrollo teórico del ETM en el dominio Álgebra nos fueron dando evidencia, adicional a la obtenida en (MONTOYADELGADILLO et al., 2014) acerca de cómo la operatoria algebraica asumió de facto un rol protagónico argumentativo en las clases, al punto de inhibir las demostraciones en geometría y otros dominios no algebraicos. Por ejemplo, en la práctica, el profesor consideraba que, en cuanto las ecuaciones planteadas tenían solución, el resultado geométrico buscado era 
correcto. Más aún, ante preguntas de sus alumnos, el PD a menudo simplemente resolvía el problema mediante algoritmos algebraicos sin poner especial atención a las propiedades relevantes del dominio fuente ni a su justificación.

Esto nos permitió verificar una vez más la fuerte influencia del álgebra en el desarrollo del ETM-personal del profesor y, por tanto, en su ETM-idóneo.

Si bien es claro que un estudio de casos no permite generalizar los resultados obtenidos a la generalidad de los PD del país, queremos enfatizar que en las clases grabadas de geometría y de probabilidades hubo un fuerte predominio de contenidos y tratamientos algebraicos y que, a pesar de la evidente potencia de las técnicas algebraicas en ellos, la falta de retorno al dominio fuente (MONTOYA; VIVIER, 2014) impidió en la práctica el desarrollo del ETM-personal de los alumnos en toda su dimensión.

En lo que se refiere a las circulaciones (MONTOYA-DELGADILLO et al., 2014), en las 60 sesiones de clase observadas se constata que los profesores debutantes activan las génesis semiótica e instrumental, en tanto que la génesis discursiva prácticamente no es desplegada: teoremas y definiciones son escasamente mencionados por los PD y quedan a nivel de fórmulas, y no hay intento de hacer un tratamiento que se ocupe de dar y/o fomentar las pruebas de los asertos que se invocan.

Nuestro estudio manifiesta que a los PD se les dificulta hacer una transposición didáctica harmónica de los contenidos geométricos y algebraicos. De hecho, parecen divididos entre las exigencias de su institución formadora y aquellas que deben poner en juego en su enseñanza: por una parte, intentan recurrir al álgebra como haría un especialista (i. e., el álgebra como instrumento), pero no consideran que el estudiante quedaría atrapado en el plano [Sem-Ins] del $\mathrm{ETM}_{\mathrm{A}}$ (algebraico) a no ser que el profesor presente explícitamente la conexión con el resultado del dominio al que se refiere la expresión algebraica en consideración -el teorema de Pitágoras se reduce a una ecuación, e.g.

\subsection{El caso de la probabilidad}

Diversos informes se refieren a la inseguridad que declararon los profesores de enseñanza básica chilenos en el dominio geometría (OCDE, 2004).

Nos pareció sin embargo conveniente procurar priorizar aquellas materias del currículo acerca de las cuales los profesores experimentaban mayor dificultad. Para ello, tomamos un curso de postgrado para profesores, constituido por 20 estudiantes, todos 
profesores en ejercicio y 17 de los cuales son PD (pero no pertenecientes al grupo en estudio). $33 \%$ de esos profesores se desempeña en el nivel básico, $52 \%$ en enseñanza media y $15 \%$ en el nivel terciario. Se les preguntó: ¿En qué materias o contenidos se siente usted más débil al momento de enseñar?. El 75\% de los encuestados declaró sentirse débil en el eje de Estadística y probabilidad; si no se considera a los de nivel terciario, este porcentaje aumenta considerablemente. La situación había sido evidenciada con otras cohortes de ese mismo postgrado, pero sin guardar un registro formal.

Lo anterior sugiere una de las razones por las cuales encontramos solo un profesor que estaba dispuesto a desarrollar el eje de Estadística y probabilidades en las clases observadas, a pesar de que esa materia está en las propuestas curriculares: en Chile, cerca de un 33\% de los contenidos obligatorios a tratar en la asignatura de Matemática en Enseñanza Media corresponden a este eje. Otra razón a considerar es que la cantidad de cursos de estadística en las mallas de formación inicial se reduce a uno o a lo sumo dos cursos, y, simplemente, la generalidad de las instituciones formadoras no contempla cursos de didáctica de estas materias.

En el período de las grabaciones, solo PD3 trató el tema de las probabilidades, en dos sesiones de clases en un $1^{\circ}$ Medio.

En la primera sesión de PD3, identificamos 4 episodios. El primero consistió en introducir intuitivamente la noción de probabilidad mediante aproximaciones al significado; esto parecía un recordatorio del tema, y los alumnos mencionaron posibilidad, resultado, oportunidad, aproximación. En el segundo episodio, el PD calcula una probabilidad, demandando a sus estudiantes, mediante ejemplos, el cálculo correspondiente. En el tercero, distingue entre fenómeno determinista y fenómeno aleatorio, todo a través de ejemplos en los cuales hace participar a sus estudiantes. Finalmente, en el cuarto episodio, escribe la regla de Laplace (sin abordar la equiprobabilidad) y expone ejercicios para su resolución, sin escribir en la pizarra. En la presentación de cada episodio usó diapositivas, las cuales contenían dibujos de dados y bolas de colores.

A continuación mostramos un extracto de la clase donde PD3 presenta la regla de Laplace (en una diapositiva) y ejemplifica para el cálculo de las probabilidades.

PD3: Bien, pasemos a la siguiente entonces. Esos son los tipos de experimentos que existen y ¿sobre qué trabaja la probabilidad entonces? Se supone que en los deterministas el resultado ya se puede conocer, pero en los aleatorios no hay una certeza de lo que pueda suceder; entonces la probabilidad mide la frecuencia con la que aparece un resultado determinado cuando se realiza, y este tiene una cantidad de sucesos determinados. ¿Cómo se calcula la probabilidad? Y esto es la regla de Laplace y se calcula en número de casos favorables dividido por el número de casos totales, y eso me va a dar la probabilidad de que 
un suceso pueda ocurrir. Por ejemplo, tenemos un ejemplo para analizar: En una caja hay 12 bolas negras y 8 rojas ¿qué probabilidad hay de sacar una bola negra?

Al: $\quad 12$ de 20

PD3: Sería. Lo primero que hay que determinar es que la probabilidad es: casos favorables partido por casos totales... Para el caso de sacar una bola negra ¿cuántos casos favorables tenemos?

Al: $\quad 12$

PD3: Son 12, cierto, porque dice sacar una bola negra ¿cuántas bolas negras tenemos?

Al: $\quad 12$

PD3: Serían 12 dividido por los casos totales. Los casos totales son todos los casos que existen dentro del experimento. Son 12 bolas negras y 8 bolas roja ¿Cuántos casos tenemos en total? 20, la probabilidad es 12 de 20. Ahora, el caso de sacar una bola roja sería: los casos favorables son 8 partido por los casos totales que son 20 y eso es nada más la probabilidad para sacar este tipo de problemas. No es complicado, solamente tengo que analizar cuántos casos totales tengo y lo que me están pidiendo, cuántos casos favorables resultan del experimento. Arriba el total de casos favorables y abajo el total de casos del experimento.

Se observa que, a pesar de la motivación que PD3 ofrece para introducir la noción probabilidad en esta clase, PD3 muestra la fórmula y luego se limita a utilizarla para realizar cálculos. No se aprecia una circulación por la génesis discursiva; nada se justifica, y rápidamente se pasa al cálculo algebraico.

Este episodio termina con ejercicios realizados con la regla de Laplace para el cálculo de una probabilidad, relativos tanto a números en un dado como a colores por sabor de un jugo. En el primer caso, se obtiene la probabilidad de que, al lanzar un dado, se obtengan, respectivamente, un número determinado, un par, un primo y la suma de dos de ellos.

PD3 impone una fórmula sin realizar la génesis discursiva. Sin embargo, realiza una circulación en el plano del descubrimiento recurriendo al artefacto simbólico fracción. Por otro lado, recurre a elementos del representamen que aluden a la fórmula, en el caso finito, como fracción " 12 dividido por 20 ", "8 partido por 20 " y finalmente como probabilidad " 12 de $20 \%$.

En la segunda clase de PD3, identificamos 3 episodios. En el primero, él muestra dos videos mediante los cuales introduce la idea de que una probabilidad puede alcanzar valores muy pequeños, "baja probabilidad que un evento suceda", y la enfatiza recurriendo a términos porcentuales; desarrolla y explica que una probabilidad puede ser muy baja y enuncia una propiedad ad hoc. El segundo episodio muestra experimentos con dos sucesos consecutivos, desviando la atención hacia el cálculo para recordar la multiplicación de fracciones. El tercer y último episodio identificado es un experimento realizado en grupos por los estudiantes en la clase, que consiste en lanzar "varias" veces una moneda al aire y consignar si sale cara o sello.

Es en el último episodio donde nos queremos detener, pues, a pesar de los esfuerzos de introducción de las nociones matemáticas involucradas y de la motivación que provee, PD3 
muestra que este experimento consiste en "comprobar la fórmula" dada en la clase en lo que se refiere al cálculo de la probabilidad de un suceso. A continuación mostramos un extracto de la clase en el cual la fórmula se vuelve la cuestión a comprobar y, por una parte, no se desarrolla una circulación apropiada en el espacio de trabajo y, por otra, no se instrumentaliza el artefacto material moneda; finalmente, a pesar de que un alumno, al igual que en el episodio anterior, da la respuesta correcta, PD3 señala que el experimento permitirá comprender la fórmula.

PD3: Bien muchachos, si me prestan atención vamos a poder hacer el experimento que nos va a permitir comprender de dónde viene la regla que nosotros utilizamos para el cálculo de probabilidad. El experimento consiste en lo siguiente: nosotros sabemos cuál es la probabilidad de que yo al lanzar una moneda me salga un sello.

Al: $\quad$ Una de dos.

PD3: Es una de dos, es un medio; si nosotros eso lo calculamos en porcentaje, tenemos un $50 \%$ de que aparezca un sello. Veamos si esto se aplica, realmente, al experimento de probabilidad. Qué es lo que voy a hacer yo con la moneda que le acabo de entregar: voy a hacer el experimento a ver si el resultado final se acerca al 50\%, vamos a ver si se acerca al 50\%. Si cumple con la regla mediante el experimento que voy a hacer. En qué consiste el experimento: voy a lanzar una moneda 50 veces; la veo [verifica si la moneda es cara o sello] y el primer lanzamiento me salió sello; luego lanzo nuevamente, segundo me sale sello y así voy marcando. Luego que haya lanzado las 50 veces y tenga mi tabla de datos, los datos que me salieron, por ejemplo lanzo la tercera vez y me sale una cara, lanzo y me sale un sello, lanzo y aquí otra vez sale una cara; después, cuando tenga los 50 lanzamientos, voy a contar cuántos sellos me salieron en el experimento, y también voy a contar cuántas caras me salieron en el experimento. Me sale un número de caras y lo divido por 50 y lo multiplico por 100, para saber qué porcentaje corresponde el experimento. Me sale la cantidad de sellos, lo divido por 50, que es la cantidad de veces que yo hice la prueba de lanzar la moneda, y luego voy a comprobar si los resultados que les dan se acercan o no se acercan al 50\%, que es la probabilidad que nosotros calculamos teóricamente... Hagan el experimento... cuando tengan los resultados me llaman para poder observar y poder hacer el cálculo de probabilidad...

[El profesor revisa los experimentos de los alumnos y comparte algunos resultados con la clase]

La situación del profesor PD3 nos parece significativa, pues nos muestra la concepción que él tiene de las probabilidades y el rol que juega la fórmula en un experimento que obedece a la ley de los grandes números, evidenciando un ETM-idóneo influenciado por un ETM-personal desprovisto de un apropiado referencial. En la trascripción, se puede observar que el alumno, antes de realizar el experimento, entrega la respuesta correcta; sin embargo PD3 confunde el representamen de la probabilidad, 1/2, con 50\%. De esta manera, la génesis semiótica no es activada apropiadamente. El artefacto material (moneda) se instrumentaliza, es realmente parte del proceso, pero ello no permite una circulación con los otros polos, puesto que el resultado es forzado por PD3 a un valor numérico dado en términos de porcentaje, a pesar de que la regla de Laplace entrega un número entre 0 y 1 , y de que el experimento no necesariamente da $1 / 2$ ya que la cantidad de sucesos es finita. No ha habido 
pregunta; la respuesta, una regla, se ha dado al inicio. Se prometió explicar de dónde viene esa respuesta, pero solo se ha hecho una suerte de argumento heurístico para esa regla. En lugar de ofrecer un fundamento a partir de la equiprobabilidad de estabilidad de las condiciones (GAUVRIT, 2014), o como señala Parzysz (2014) usar simulación informática de experiencias aleatorias; a cambio PD3 ha explicado cómo se calcula un porcentaje; de tal manera, se ha algebrizado el asunto, y a la vez se ha perturbado la génesis semiótica.

\subsection{El caso de las ecuaciones}

En el periodo de observación, dos de los 6 PD observados, PD5 y PD1, trabajaron las ecuaciones de primer grado. Nos propusimos no hacer un estudio comparativo entre los PD, pero estudiamos los currículos de las universidades de procedencia, para obtener información y aproximarnos al ETM de referencia.

El caso que sigue corresponde a la manera de desplegar el ETM-idóneo de PD5, diferente a la del resto de los PD, los cuales exhiben en esto un fuerte trabajo algorítmico.

Como se verá a continuación, PD5 plantea una ecuación dejando a los estudiantes en situación de ruptura, en la cual la algoritmia no es suficiente para establecer la solución de la ecuación. Para ello, PD5 lleva a la pizarra las respuestas de tres grupos distintos (ver figura 2) y las confronta frente al grupo curso. Aprovechando que ninguna de las soluciones ofrecidas da un resultado correcto, PD5 hace preguntas que llevan a los estudiantes a encontrar distintos números que satisfacen la ecuación y a concluir que hay "muchas soluciones". Nosotros consideramos que PD5 activa la génesis discursiva apropiadamente recurriendo al referencial y permitiendo la circulación entre los polos del ETM, como se observa en lo que sigue.

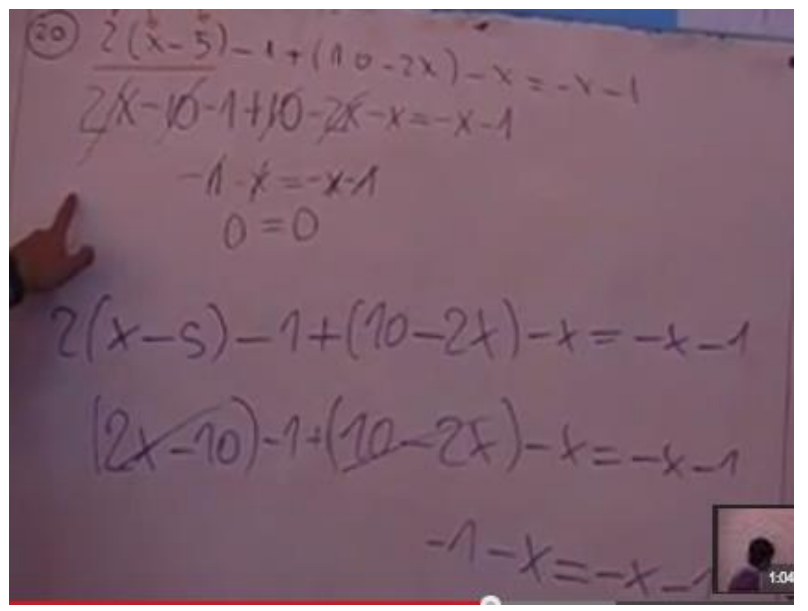

Figura 1 - PD5 revisando resultados de los alumnos 
Desde el punto de vista del ETM-idóneo, se observa la atención que despliega PD5 para exponer el caso de infinitas soluciones. Identificamos este episodio como uno de los pocos ejemplos de activación de la génesis discursiva que pudimos observar, y naturalmente es también importante consignar que activó las otras génesis en este dominio. En cuanto a la génesis semiótica, PD5 utiliza los signos de ecuación y de variable, y promueve la visualización solo a través de estos elementos del representamen, sin pasar por las gráficas, que normalmente ayudan a robustecer el significado de este tipo de soluciones. Ver también MONTOYA-DELGADILLO; MENA-LORCA; MENA-LORCA, 2014).

\section{Conclusiones}

Explicitamos aquí algunas conclusiones, parciales respecto de nuestro proyecto general.

\subsection{Los profesores evaden algunos contenidos}

Los contenidos que los PD examinados tratan en las clases muestran que ellos no abordan temas como los del eje de Estadística y probabilidades; también evitan temas tales como la resolución de problemas y la modelación, que en la actualidad son claramente exigibles en los currículos chilenos.

La formación inicial de la mayoría de los PD chilenos no prepara para trasponer el saber de la estadística y de las probabilidades. Para profesores de enseñanza básica la matemática se incluye en cursos de metodología o de "didáctica general", que abordan todas las disciplinas escolares en conjunto; para profesores de enseñanza media hay cursos básicos de estadística y probabilidades en un porcentaje muy escaso del currículo universitario (y considerablemente menor que el que supondría su presencia en el currículo escolar). Para ambos tipos de profesores, no hay cursos de didáctica de la estadística. La situación internacional al respecto es parecida (ESTRELLA, 2016; ESTRELLA; OLFOS; MENALORCA, 2015).

A pesar de la creciente importancia de las ideas de modelación y de resolución de problemas en lo que se suele llamar matemáticas para la vida, en Chile se las ha incluido solo recientemente en el currículo. Además, la formación inicial no las incluye como un saber que 
se debe transponer; de allí que los PD las consideren como una aplicación directa de un contenido en desarrollo o ya desarrollado, perdiéndose gran parte de su riqueza.

La algebrización es una otra manera de evadir un contenido, observada en los PD del estudio. Consiste en cambiar a otro dominio y no retornar al dominio origen. Por ejemplo, al enseñar cuestiones de geometría reiteradamente recurren al álgebra, como dominio de resolución, pero no siempre parecen conscientes de la necesidad de retornar al dominio de la geometría para dar respuesta y coherencia a la tarea realizada. Esto se agudiza en geometría analítica, a la que se reduce a manejo operatorio -asociar expresiones cuadráticas a las cónicas, o similares. En Estadística y probabilidades, ese deslizamiento hacia el álgebra desvirtúa el dominio y lo convierte en aplicación de fórmulas que pierden su significado original. De tal manera, el PD no toma conciencia de que debe desarrollar y explotar en plenitud un dominio específico de acuerdo al contenido que quiere tratar; en un dominio distinto del álgebra, podría requerir de artefactos del álgebra y mantener momentáneamente a esta a nivel de herramienta de aquel, y retornar luego al dominio original.

\subsection{La demostración y su trasposición}

Los PD tienen dificultades para activar la génesis discursiva. Una de las razones para ello está en la formación inicial, en la cual no se enseña a transponer la demostración; es decir, en la génesis discursiva del ETM-personal del estudiante en formación, la única prueba válida es la demostración de los matemáticos -Montoya-Delgadillo (2014) ya lo había evidenciado en el dominio de la geometría. El seguimiento de los profesores debutantes observados ha mostrado que en general ellos no activan esa génesis discursiva y por consiguiente tampoco el plano semiótico-discursivo, [Sem-Disc] (ver KUZNIAK; RICHARD, 2014). Ello ocurre ya sea porque en su ejercicio profesional se remiten a introducir fórmulas cuya aplicación posteriormente exigen sin mayor fundamento, o bien porque la construcción en la génesis instrumental cumple el rol de prueba, pues el resultado, en cuanto existe o se puede conseguir con algún procedimiento, es la única justificación o validación que ofrecen -la obtención de la solución de una ecuación ante un problema geométrico, e. g.

En general, en estos profesores, la circulación se da en el plano [Sem-Inst] (ver KUZNIAK; RICHARD, 2014), conformado por las génesis semiótica e instrumental, ya que solo se activan dichas génesis. El PD que trabajó con el artefacto material moneda logró una 
verdadera circulación en el plano [Sem-Inst]; sin embargo, ese mismo profesor, que usó videos para introducir la idea de que un evento es poco probable, no activó la génesis instrumental.

\subsection{La estabilidad epistemológica}

Según hemos señalado antes, el PD reacciona en la clase en dos dimensiones: la profesional, y la personal como matemático. En este sentido podemos concluir que su epistemología es inestable. En efecto, su ETM-idóneo está determinado en primera instancia por el ETM de referencia recibido en su formación.

En un dominio específico, la formación inicial recibida, en los cursos disciplinares, puede estar focalizada en lo discursivo y ubicada en el paradigma más avanzado. Ello no obstante, la circulación entre las componentes y la presencia de los distintos planos verticales es ostensible, y las técnicas utilizadas en cada uno de ellos son claras, como así también son claros los procesos algorítmicos empleados, los que cumplen un rol de artefacto simbólico que no desvirtúa el dominio desarrollado. En otras palabras, hay, en principio, una posibilidad de desarrollar un ETM personal.

Sin embargo, enfrentado a la tarea de transponer los saberes, se encuentra con que no ha tenido experiencia para activar la génesis discursiva, pues la institución formadora no le dio elementos suficientes para efectuar su transposición. De tal manera, el profesor debutante concluye, luego de su experiencia, que los procesos de demostración no son viables en el nivel escolar del cual se ocupa y, de este modo, se privilegia la operatoria. Por tanto, su desempeño en el aula está tensionado entre lo que piensa como matemático y lo que piensa como profesor en un paradigma no siempre explícito para él.

Hay, por tanto, una amplia brecha entre la construcción del ETM personal y el ETM idóneo de un profesor debutante, ya que en su formación inicial no encuentra las herramientas que requiere. El marco teórico del ETM, que permite no solo identificar el rol de saberes que ayudan a la construcción de otro saber en particular, sino también ofrecer la forma de abordar el proceso de transposición y abordar la problemática antes señalada; ya que preocuparse por la activación de las génesis y crear circulaciones apropiadas permiten la compleción de un ETM idóneo que posibilita atender a las necesidades tanto de los estudiantes como del profesor en su rol. 


\section{Agradecimientos}

Este trabajo fue financiado parcialmente por los proyectos FONDECYT $\mathrm{N}^{\circ} 1110988$ y $\mathrm{N}^{\circ} 1151376$.

\section{Referencias}

ESTRELLA, S. Comprensión de la media por profesores de educación primaria en formación continua. REDIE, Revista Electrónica de Investigación Educativa, Baja California, v. 18, n. 1, p. 122, ene. 2016.

ESTRELLA, S.; OLFOS, R.; MENA-LORCA, A. El Conocimiento Pedagógico del Contenido de Estadística en Profesores de Primaria. Revista Educação e Pesquisa, Sao Paulo, v. 41, n. 2, p. 477 493, abr./jun.2015.

GAUVRIT, N. A propos du «biais d'équiprobabilité ». Recherches en didactique des mathématiques, Grenoble, v. 33, n. 2, p. 163-182, 2013.

HOUDEMENT, C.; KUZNIAK, A. Autours des stratégies utilisées pour former les maîtres du premier degré en mathématiques. Recherches en Didactique des Mathématiques, Grenoble, v. 16, n. 3, p. 289-321, 1996.

HOUDEMENT, C.; KUZNIAK, A. Paradigmes géométriques et enseignement de la géométrie. Annales de Didactique et de Sciences Cognitives, Strasbourg, v. 11, p. 175-193, 2006.

KUZNIAK, A. l'espace de travail mathématique et ses genèses. Annales de Didactique et de Sciences Cognitives, Strasbourg, v. 16, p. 9-24, 2011.

KUZNIAK, A.; RICHARD, P. R. Espacios de trabajo matemático. Puntos de vista y perspectivas. Revista Latinoamericana de Investigación en Matemática Educativa, RELIME, México, v. 17, n. 4-I, p. 5-15, dic. 2014.

MONTOYA-DELGADILLO, E. El proceso de prueba en el espacio de trabajo geométrico: profesores en formación inicial. Revista Enseñanza de las Ciencias, Barcelona, v. 32, n. 3, p. 227-247, nov. 2014.

MONTOYA-DELGADILLO, E.; MENA-LORCA, A.; MENA-LORCA, J. Circulaciones y génesis en el espacio de trabajo matemático. Revista Latinoamericana de Investigación en Matemática Educativa, RELIME, México, v. 17, n. 4-I, p. 181-1997, dic. 2014.

MONTOYA-DELGADILLO, E.; VIVIER, L. Les changements de domaine de travail dans le cadre des Espaces de Travail Mathématique, Annales de Didactique et de Sciences Cognitives, Strasbourg, v. 19, p. 73-101, 2014.

ORGANIZACIÓN DE LAS NACIONES UNIDAS PARA LA EDUCACIÓN, LA CIENCIA Y LA CULTURA - UNESCO. Antecedentes y criterios para la elaboración de políticas docentes en américa latina y el caribe. Santiago: UNESCO, 2013.

ORGANIZACION PARA COOPERACION Y DESARROLLO ECONOMICO (OCDE). Revisión de Políticas Nacionales de Educación. 2004. Disponible en <http://www.oecdilibrary.org/education/revision-de-politicas-nacionales-de-educacion-chile_9789264021020es;jsessionid=9gbdr033d0sq2.x-oecd-live-02>. Acceso en : 14 nov. 2007. 
PARZYSZ, B. Évolution du travail mathématique dans l'enseignement des probabilités en France depuis 1980. In: GÓMEZ-CHACÓN, I. M. et. al (Ed.). Mathematical Working Space, Proceedings Fourth ETM Symposium... Madrid: Publicaciones del Instituto de Matemática Interdisciplinar, Universidad Complutense de Madrid, 2015. p. 39-50. Disponible en <

http://www.mat.ucm.es/imi/ETM4/francais.html>. Acceso en: 14 nov. 2015.

PORLÁN, R.; RIVERO, A.; DEL POZO, M. Conocimiento profesional y epistemología de los profesores, ii: estudios empíricos y conclusiones. Revista Enseñanza de las Ciencias, Barcelona, v. 16, n. 2, p. 271-288, jun.1998.

Submetido em Julho de 2015. Aprovado em Setembro de 2015. 